\title{
Analysis of major ginsenosides in various ginseng samples
}

\author{
Dong Gu Lee ${ }^{1} \cdot$ Ju Sung Lee ${ }^{1} \cdot$ Kyung-Tack Kim² ${ }^{2}$ Hyun Young Kim ${ }^{3}$ Sanghyun Lee ${ }^{1}$
}

Received: 15 November 2018 / Accepted: 7 February 2019 / Published Online: 31 March 2019

(C) The Korean Society for Applied Biological Chemistry 2019

\begin{abstract}
The contents of major ginsenosides (ginsenosides $\mathrm{Rb}$, ginsenoside $\mathrm{Rc}$, ginsenoside $\mathrm{Rd}$, ginsenoside $\mathrm{Re}$, ginsenoside $\mathrm{Rf}$, and ginsenoside $\mathrm{Rg} 1$ ) in ginseng cultivated in different areas in Korea, ginseng that underwent different cultivation processes and ages, and ginseng cultivated in different countries were determined using high-performance liquid chromatography equipped with UV/VIS detector. Ginsenoside Rc was the most abundant ginsenoside in all different ginseng samples. The highest total concentration of major ginsenosides was found in the ginseng cultivated in Jinan $(0.931 \mathrm{mg} / \mathrm{g})$ and 4-year grown red ginseng $(1.785 \mathrm{mg} / \mathrm{g})$. Major ginsenosides were the most abundant in Korean ginseng $(1.264 \mathrm{mg} / \mathrm{g})$, compared to those in Chinese and American ginseng. The results of this study showed the different contents of major ginsenosides in the ginseng samples tested and emphasized which sample could contain high yield of ginsenosides.
\end{abstract}

Keywords Ginsenoside $\cdot$ Panax species $\cdot$ Quantitative analysis

\section{Introduction}

Panax ginseng, more commonly known as Korean ginseng, is a traditional herbal medicine that has been widely used in Asian and Western countries for more than 2000 years. Ginseng is a perennial

Sanghyun Lee $(\triangle)$

E-mail: slee@cau.ac.kr

${ }^{1}$ Department of Plant Science and Technology, Chung-Ang University, Anseong 17546, Republic of Korea

${ }^{2}$ Korea Food Research Institute, Wanju 55365, Republic of Korea

${ }^{3}$ Department of Food Science, Gyeongnam National University of Science and Technology, Jinju 52725, Republic of Korea

This is an Open Access article distributed under the terms of the Creative Commons Attribution Non-Commercial License (http://creativecommons. org/licenses/by-nc/3.0/) which permits unrestricted non-commercial use, distribution, and reproduction in any medium, provided the original work is properly cited. plant, which belongs to the family Araliaceae. It is known to alleviate various human diseases, such as diabetes mellitus, depression, aging, nausea, inflammation, pulmonary diseases, and cardiac ischemia [1-4]. Other species from the same genus, such as $P$. notoginseng (Chinese ginseng) and $P$. quinquefolius (American ginseng), share the same health benefits with Korean ginseng [5,6]. These ginseng species contain various bioactive compounds, such as acidic polysaccharides, polyphenols, phytosterols, polyacetylenes, and ginseng saponins, which can be involved in ginseng's various health benefits $[7,8]$. Among these components, ginseng saponins, more popularly known as ginsenosides, are the most characterized based on their biological activity [9].

Ginsenosides are unique to ginseng species, and their pharmacological mechanisms of action in cardiovascular diseases, diabetes mellitus, various cancers, and stress, as well as their immunostimulatory, and anti-inflammatory activities have been reported in several studies to date [4]. More than 50 ginsenosides have been already identified; among them, 6 major ginsenosides (Rb1, Rb2, Rc, Rd, Re, and Rg1) have been found to comprise $90 \%$ of the total ginsenoside content of $P$. ginseng [10]. These ginsenosides are naturally present in this plant.

Scientists aim to develop nanoparticles of the major components of ginseng. The use of nanoparticle delivery system to target diseased cells has been gaining popularity for the last few years. For instance, encapsulation of unmethylated cytosine-phosphateguanosine oligodeoxynucleotides in nanoparticles has been proven effective in activating the immune system for the treatment of cancers, infectious diseases, and allergies [11-13].

As part of our continued research on ginseng and its potential applications in functional food research, in this study, we aimed to evaluate ginsenoside contents in different ginseng varieties, ginseng at different cultivation ages, and ginseng cultivated in different countries.

\section{Materials and Methods}

Plant materials

Ginseng samples grown in different areas in Korea (Geumsan, 
Yeongpu, and Jinan), produced using different manufacturing methods (red, straight, and white ginseng) supplied by Korea Food Research Institute, harvested at different cultivation ages (4, 5, and 6 years), Jinan, Korea, and obtained from different countries (Korea, China, and America) of 5-year grown samples were tested. Briefly, the cultivation conditions of Korean ginseng used in the experiment were as follows: annual average temperature and average precipitation were expected $4-10^{\circ} \mathrm{C}$ and $800-900$ $\mathrm{mm}$, respectively. Ginseng requires 80 percent shade to thrive and prefers a deep rich loamy soil. The roots can be harvested once in the fall after the leaves die.

\section{Reagents and instruments}

High-performance liquid chromatography (HPLC) chromatograms were recorded on a Waters 1525 Binary HPLC pump equipped with a Waters 2489 UV/VIS detector (Miami, CA, USA). A SunFire C-18 column $(2.1 \times 50 \mathrm{~mm}, 5 \mu \mathrm{m})$ was used for chromatographic separation. All reagents, including methanol $(\mathrm{MeOH})$, acetonitrile, and chloroform $\left(\mathrm{CHCl}_{3}\right)$, were of HPLC grade.

\section{Preparation of standard and samples for HPLC analysis} For analysis of major ginsenosides (Figs. 1 and 2), $200 \mathrm{~g}$ of each ginseng sample was extracted with ethanol $(\mathrm{EtOH} ; 3 \times 100 \mathrm{~mL})$ under reflux and evaporated in vacuo. One milligram of each extracted sample was dissolved in $1 \mathrm{~mL}$ of $\mathrm{MeOH}$. For injection, sample solutions were filtered through a Whatman $0.45-\mu \mathrm{m}$ polyvinylidene difluoride syringe filter (Cat No. 6779 1304, Piscataway, NJ, USA). The filtrate was used for HPLC analysis.

\section{HPLC conditions}

For qualitative and quantitative analysis of major ginsenosides isolated from the roots of $P$. ginseng, each sample was prepared as described above. For identification and quantification of ginsenoside contents by HPLC, distilled water and acetonitrile (water: ACN, $v / v)$ were used as the mobile phase. Gradient elution was used$95 \%$ water initially, then ACN was increased in linear gradient to water : $\mathrm{ACN}=65: 35$ for $35 \mathrm{~min}$, and finally 20:80 for $40 \mathrm{~min}$. The flow rate was $1 \mathrm{~mL} / \mathrm{min}$. The detection wavelength was 204 $\mathrm{nm}$, and sample injection volume was $10 \mu \mathrm{L}$. All solvents used in HPLC analysis were degassed before use. All injections were performed in triplicate.

\section{Calibration curves}

A stock solution $(1 \mathrm{mg} / \mathrm{mL})$ of major ginsenosides isolated from $P$. ginseng was prepared in $\mathrm{MeOH}$. The contents of the analytes were determined from the corresponding calibration curves. The concentrations of flavone derivatives $(\mathrm{X} \mu \mathrm{g} / 10 \mu \mathrm{L})$ were calculated based on the corresponding peak areas $(\mathrm{Y})$, and expressed as the mean values $(n=3) \pm$ standard deviations.

\section{Statistical analysis}

All statistical analysis was done with SAS program (SAS Institute
Inc., Cary, NC, USA). Analysis of variance was used to determine the main and interaction effects. The least significant difference test was applied for determining the mean separation at $p=0.05$.

\section{Results and Discussion}

Major ginsenoside content in the roots of ginseng samples was analyzed by HPLC. Major ginsenosides (Figs. 1, 2) were previously isolated from $P$. ginseng roots and named as ginsenoside Rb1 (1), ginsenoside Rc (2), ginsenoside Rd (3), ginsenoside Re (4), ginsenoside Rf (5), and ginsenoside Rg1 (6) [14]. HPLC separation of ginsenosides for qualitative and quantitative analysis was performed using a reverse-phase system. The chromatograms of major ginsenosides are shown in Fig. 3. Among the major ginsenosides, ginsenoside Rc was the most abundant in all ginseng samples tested. Ginsenoside Rc is considered the main ginsenoside that contributes to the antioxidant activities of ginseng. Ginsenoside Rc was shown to induce the overexpression of catalase, which inhibited the production of reactive oxygen species in human embryonic kidney $293 \mathrm{~T}$ cells [15].

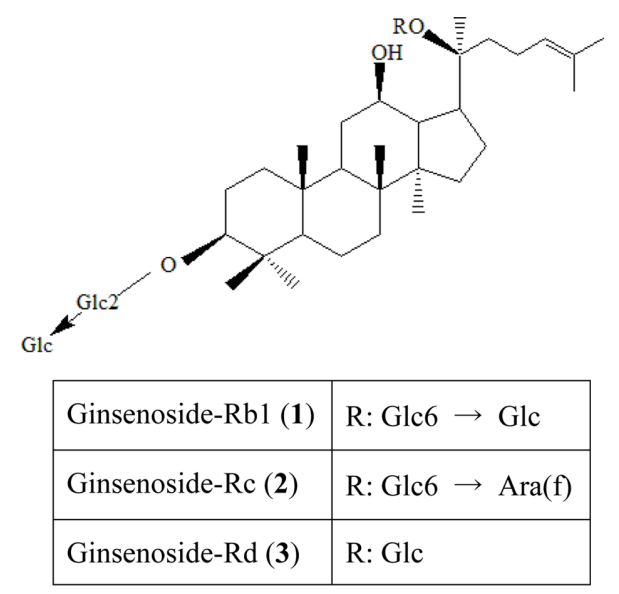

Fig. 1 Chemical structures of protopanaxadiol types

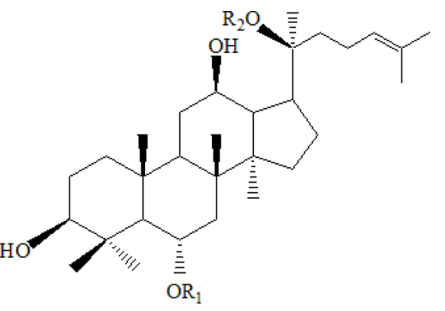

\begin{tabular}{|l|l|l|}
\hline Ginsenoside-Re (4) & $\mathrm{R}_{1}: \mathrm{Glc} 2 \rightarrow \mathrm{Rha}$ & $\mathrm{R}_{2}: \mathrm{Glc}$ \\
\hline Ginsenoside-Rf (5) & $\mathrm{R}_{1}: \mathrm{Glc} 2 \rightarrow \mathrm{Glc}$ & $\mathrm{R}_{2}: \mathrm{H}$ \\
\hline Ginsenoside-Rg1 (6) & $\mathrm{R}_{1}: \mathrm{Glc}$ & $\mathrm{R}_{2}: \mathrm{Glc}$ \\
\hline
\end{tabular}

Fig. 2 Chemical structures of protopanaxatriol types 
(A)

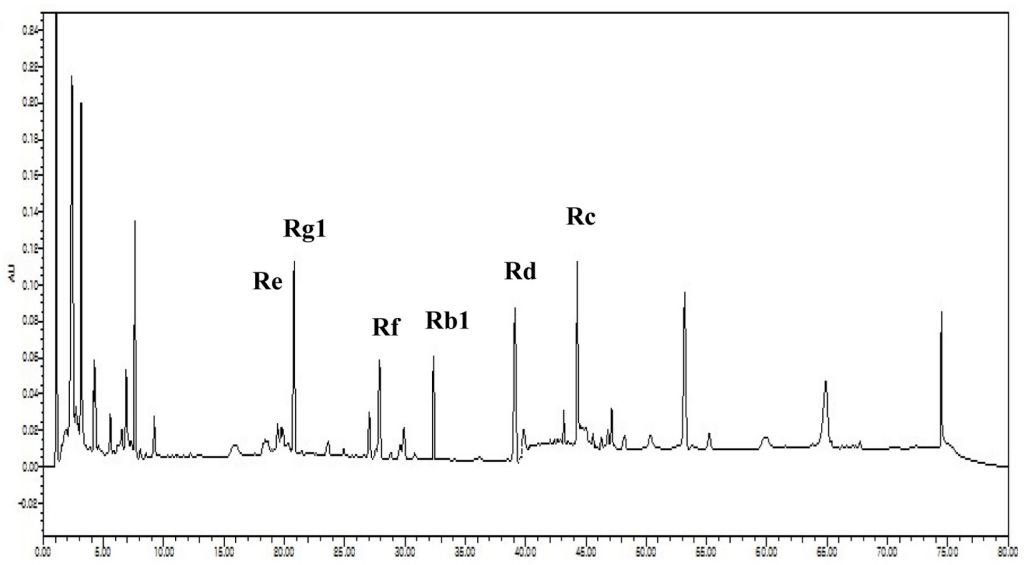

(B)

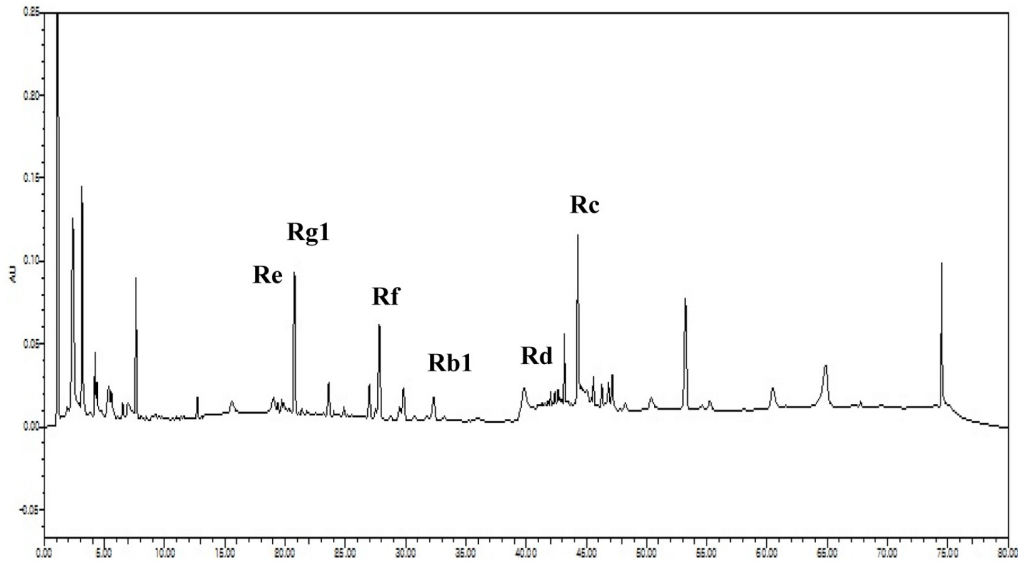

(C)

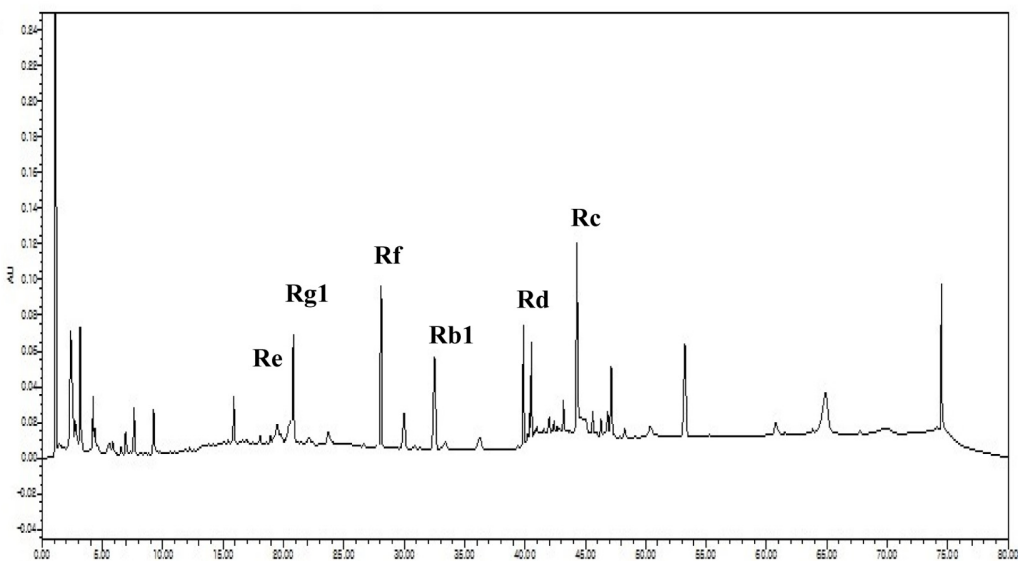

Fig. 3 HPLC chromatograms of the EtOH extracts of ginseng species obtained from Geumsan (A), 4-year grown red ginseng (B), and ginseng cultivated in America (C)

Compounds 1-6 were assayed in ginseng samples grown in different areas in Korea (Table 1). Results showed that ginseng cultivated in Jinan exhibited the highest ginsenoside Rc content $(0.495 \mathrm{mg} / \mathrm{g})$ and the highest total major ginsenoside content $(0.931 \mathrm{mg} / \mathrm{g})$. A recent study using ginseng samples cultivated in Geumsam, Yeongju, and Jinan showed that ginseng samples from Jinan also had the highest phytosterol content [16]. Phytosterol is another bioactive compound present in ginseng species. It is known to exhibit anti-inflammatory, antifungal, antibacterial, antitumor, and hypocholesterolemic activities [6]. Moreover, ginseng obtained from Jinan had higher non-saponin acidic polysaccharide content than that in ginseng obtained from Geumsam [17]. These results suggested that ginseng grown in Jinan of different environmental condition might be a better source of bioactive compounds, compared to that grown in Yeonju and Geumsam.

Regarding the major ginsenoside content in ginseng samples cultivated using different production processes and cultivation 
Table 1 Contents of major ginsenosides in ginseng species cultivated in different areas in Korea

\begin{tabular}{lccccccc}
\hline \hline \multirow{2}{*}{ Sample } & \multicolumn{7}{c}{ Content $(\mathrm{mg} / \mathrm{g})$} \\
\cline { 2 - 8 } & $\mathbf{1}$ & $\mathbf{2}$ & $\mathbf{3}$ & $\mathbf{4}$ & $\mathbf{5}$ & $\mathbf{6}$ & Total \\
\hline Geumsan & $0.110 \mathrm{hij}$ & $0.260 \mathrm{f}$ & $0.046 \mathrm{o}$ & $0.123 \mathrm{~g}$ & $0.074 \mathrm{k}$ & $0.115 \mathrm{~h}$ & $0.728 \mathrm{c}$ \\
Yeongju & $0.124 \mathrm{~g}$ & $0.312 \mathrm{e}$ & $0.049 \mathrm{no}$ & $0.122 \mathrm{~g}$ & $0.066 \mathrm{l}$ & $0.115 \mathrm{~h}$ & $0.788 \mathrm{~b}$ \\
Jinan & $0.114 \mathrm{hi}$ & $0.495 \mathrm{~d}$ & $0.052 \mathrm{mn}$ & $0.109 \mathrm{ij}$ & $0.056 \mathrm{~m}$ & $0.105 \mathrm{j}$ & $0.931 \mathrm{a}$ \\
\hline
\end{tabular}

Means accompanied by the same letters are not significantly different in least significant difference test at $p=0.05$

Table 2 Contents of major ginsenosides in ginseng species obtained by different production processes and cultivation years

\begin{tabular}{|c|c|c|c|c|c|c|c|}
\hline \multirow{2}{*}{ Sample } & \multicolumn{7}{|c|}{ Content (mg/g) } \\
\hline & 1 & 2 & 3 & 4 & 5 & 6 & Total \\
\hline 4-yr Red & $0.337 \mathrm{n}$ & $0.696 \mathrm{~h}$ & $0.137 \mathrm{v}-\mathrm{y}$ & $0.244 p$ & $0.142 \mathrm{vwx}$ & $0.229 \mathrm{pq}$ & $1.785 \mathrm{a}$ \\
\hline 5-yr Red & $0.239 \mathrm{p}$ & $0.617 \mathrm{i}$ & $0.093 \mathrm{v}-\mathrm{y}$ & $0.162 \mathrm{p}$ & $0.101 \mathrm{vwx}$ & $0.153 \mathrm{pq}$ & $1.608 \mathrm{~b}$ \\
\hline 6-yr Red & $0.272 \mathrm{o}$ & $0.676 \mathrm{~h}$ & $0.097 a-c$ & 0.171 tuv & $0.100 \mathrm{z}-a$ & 0.163 tuv & $1.479 \mathrm{c}$ \\
\hline 4-yr Straight & $0.078 b-e$ & $0.366 \mathrm{~m}$ & $0.052 e-g$ & $0.075 b-f$ & $0.042 \mathrm{~g}$ & $0.072 c-f$ & $0.685 \mathrm{~h}$ \\
\hline 5-yr Straight & $0.151 \mathrm{uvw}$ & $0.324 \mathrm{n}$ & $0.067 \mathrm{~d}-\mathrm{g}$ & 0.160 tuv & $0.092 a-d$ & $0.149 \mathrm{uvw}$ & $0.943 \mathrm{f}$ \\
\hline 6-yr Straight & $0.097 a-c$ & $0.406 f g$ & $0.049 f g$ & $0.126 \mathrm{w}-\mathrm{z}$ & $0.077 b-e$ & $0.119 \mathrm{x}-a$ & $0.874 \mathrm{~g}$ \\
\hline 4-yr White & $0.274 \mathrm{o}$ & $0.512 \mathrm{j}$ & $0.099 \mathrm{z}-c$ & $0.224 \mathrm{pq}$ & $0.122 \mathrm{y}-a$ & $0.209 \mathrm{qr}$ & $1.440 \mathrm{~d}$ \\
\hline 5-yr White & $0.238 \mathrm{p}$ & $0.631 \mathrm{i}$ & $0.100 \mathrm{z}-b$ & $0.194 \mathrm{rs}$ & $0.101 \mathrm{z}-b$ & $0.183 \mathrm{rst}$ & $1.447 \mathrm{~d}$ \\
\hline 6-yr White & $0.158 \mathrm{tvu}$ & $0.468 \mathrm{k}$ & $0.069 d-g$ & 0.157 tuv & $0.084 b-d$ & $0.148 \mathrm{uvw}$ & $1.084 \mathrm{e}$ \\
\hline \multicolumn{8}{|l|}{ Significance } \\
\hline Type & $* * * *$ & $* * * *$ & $* * * *$ & $* * * *$ & $* * * *$ & $* * * *$ & $* * * *$ \\
\hline Age & $* * * *$ & NS & $* * * *$ & $* * * *$ & $* * * *$ & $* * * *$ & $* * * *$ \\
\hline Type×Age & $* * * *$ & $* * * *$ & $* * * *$ & $* * * *$ & $* * * *$ & $* * * *$ & $* * * *$ \\
\hline
\end{tabular}

Means accompanied by the same letters are not significantly different in least significant difference test at $p=0.05$. The order of mean difference was first used in normal font and then italic font in order

NS, or $* * * *$ Nonsignificant or Significant at $p \leq 0.05,0.01,0.001$, or 0.0001 , respectively

Table 3 Contents of major ginsenosides in ginseng species cultivated in different countries

\begin{tabular}{lccccccc}
\hline \hline \multirow{2}{*}{ Sample } & \multicolumn{7}{c}{ Content $(\mathrm{mg} / \mathrm{g})$} \\
\cline { 2 - 8 } & $\mathbf{1}$ & $\mathbf{2}$ & $\mathbf{3}$ & $\mathbf{4}$ & $\mathbf{5}$ & $\mathbf{6}$ & Total \\
\hline P. ginseng & $0.238 \mathrm{~g}$ & $0.631 \mathrm{~d}$ & $0.100 \mathrm{j}$ & $0.193 \mathrm{~h}$ & $0.101 \mathrm{j}$ & $0.101 \mathrm{j}$ & $1.264 \mathrm{a}$ \\
P. notoginseng & $0.016 \mathrm{1}$ & $0.468 \mathrm{f}$ & $0.154 \mathrm{i}$ & $0.205 \mathrm{~h}$ & $0.104 \mathrm{j}$ & $0.192 \mathrm{~h}$ & $1.139 \mathrm{~b}$ \\
P. quinquefolium & $0.042 \mathrm{k}$ & $0.569 \mathrm{e}$ & $0.104 \mathrm{j}$ & $0.143 \mathrm{i}$ & $0.110 \mathrm{j}$ & $0.136 \mathrm{i}$ & $1.104 \mathrm{c}$ \\
\hline
\end{tabular}

Means accompanied by the same letters are not significantly different in least significant difference test at $p=0.05$

years (Table 2), 4-year grown red ginseng exhibited the highest ginsenoside Rc content $(0.696 \mathrm{mg} / \mathrm{g})$ and the highest total major ginsenoside content $(1.785 \mathrm{mg} / \mathrm{g})$. Straight and white ginsengs of 5-year grown samples exhibited the highest total major ginsenoside content (Table 2). Previous studies showed that ginsenosides isolated from 4-year grown red ginseng rather than 6-year grown red ginseng could be used for the development of good quality products $[18,19]$. Red ginseng is prepared by heating fresh ginseng roots at $95-100{ }^{\circ} \mathrm{C}$ for $2-3 \mathrm{~h}$ [20]. Heat transforms the naturally occurring ginsenosides into their derivatives, such as ginsenoside Rg2, Rg6, F4, 20(E)-F4, and Rh1 [21]. However, red ginseng used in this study still contained higher major ginsenoside content than that in white ginseng, which did not undergo extreme heating. This suggested that red ginseng might contain more stable major ginsenosides. Dehydration might result in concentrated ginsenosides.

Korean ginseng from Jinan exhibited the highest ginsenoside Rc content $(0.631 \mathrm{mg} / \mathrm{g})$, compared to that in Chinese and American ginseng (Table 3). Korean ginseng also contained the highest total ginsenoside content $(1.264 \mathrm{mg} / \mathrm{g})$. A previous study using different parts of $P$. quinquefolius L. (American ginseng) suggested that ginsenoside Rb1 and Rc contents were affected by the location where it was grown, whereas ginsenoside Rg1 and Rd contents were affected by both the sample genotype and location [5]. This might also be true for other ginseng species, which could explain the difference in the major ginsenoside contents in the different ginseng samples examined in this study. Location affects ginsenoside contents by environmental conditions such as climate condition and different soil elements or properties.

Our study confirmed that the six major ginsenosides previously 
isolated from $P$. ginseng were also present in all the ginseng samples investigated in this study. Among the major ginsenosides present in ginseng cultivated in different parts of Korea, ginseng cultivated using different processes and cultivation ages, and different species of ginseng, ginsenoside Rc consistently exhibited the highest yield. Ginseng cultivated in Jinan had the highest ginsenoside content, compared to that cultivated in Geumsam and Yeongju, whereas 4-year grown red ginseng showed the highest total ginsenoside content. Finally, Korean ginseng exhibited the highest total ginsenoside content among the different ginseng species tested. The highest total concentration of major ginsenosides was found in the ginseng cultivated in Jinan $(0.931 \mathrm{mg} / \mathrm{g})$ and 4year grown red ginseng $(1.785 \mathrm{mg} / \mathrm{g})$. This difference in ginsenoside content could be attributed to the difference in environmental conditions and genotype. Taken together, the present study highlighted which variety, production process, and species of ginseng could be used to obtain the highest major ginsenoside yield.

Acknowledgments This research was supported by the grants of Korea Food Research Institute, Republic of Korea.

\section{References}

1. Furukawa T, Bai C-X, Kaihara A, Ozaki E, Kawano T, Nakaya Y, Awais M, Sato M, Umezawa Y, Kurokawa J (2006) Ginsenoside Re, a main phytosterol of Panax ginseng, activates cardiac potassium channels via a nongenomic pathway of sex hormones. Mol Pharmacol 70: 1916-1924

2. Lee SA, Bae B-S, Park H-W, Ahn N-G, Cho B-G, Cho Y-L, Kwak Y-S (2015) Characterization of Korean red ginseng (Panax ginseng Meyer): History, preparation method, and chemical composition. J Ginseng Res 39: 384-391

3. Chung I-M, Lim J-J, Ahn M-S, Jeong H-N, An T-J (2016) Comparative phenolic compound profiles and antioxidative activity of the fruit, leaves, and roots of Korean ginseng (Panax ginseng Meyer) according to cultivation years. J Ginseng Res 40: 68-75

4. Kim JH, Yi YS, Kim MY, Cho JY (2017) Role of ginsenosides, the main active components of Panax ginseng, in inflammatory responses and diseases. J Ginseng Res 41: 435-443

5. Zhang K, Wang X, Ding L, Li J, Qu CL, Chen LG, Jin HY, Zhang HQ (2008) Determination of seven major ginsenosides in different Panax quinquefolius L. (American ginseng) with different ages. Chem Res Chinese Universities 24: 707-711

6. Lee CH, Kim JH (2014) A review on the medicinal potentials of ginseng and ginsenosides on cardiovascular diseases. J Ginseng Res 38: 161-166

7. Yang W, Qiao X, Li K, Fan J, Bo T, Guo D-A, Ye M (2016)
Identification and differentiation of Panax ginseng, Panax quinqefolium, and Panax notoginseng by monitoring multiple diagnostic chemical markers. Acta Pharm Sin B 6: 568-575

8. Shi X-J, Yang W-Z, Qiu S, Yao C-I, Shen Y, Pan H-Q, Bi Q-R, Yang M, Wu W-Y, Guo D-A (2017) An in-source multiple collision-neutral loss filtering based nontargeted metabolomics approach for the comprehensive analysis of malonyl-ginsenosides from Panax ginseng, P. quinquefolius, and $P$. notoginseng. Anal Chim Acta 952: 59-70

9. Ru W, Wang D, Xu Y, He X, Sun Y-E, Qian L, Zhou X, Qin Y (2015) Chemical constituents and bioactivities of Panax ginseng (C.A. Mey.). Drug Discov Ther 9: 23-32

10. Park SE, Na CS, Yoo SA, Seo SH, Son HS (2017) Biotransformation of major ginsenosides in ginsenoside model culture by lactic acid bacteria. $\mathrm{J}$ Ginseng Res 41: 36-42

11. Hanagata N (2012) Structure-dependent immunostimulatory effect of CpG oligodeoxynucleotides and their delivery system. Int J Nanomed 7: 2181-2195

12. Jang H-E, Jung H, Mok H (2017) Cleavable conjugation of CpG oligodeoxynucleotides onto microparticles for facile release and cytokine induction in macrophages. Appl Biol Chem 60: 321-326

13. Castro-Aceituno V, Ahn S, Simu SY, Singh P, Mathiyalagan R, Lee HA, Yang DC (2016) Anticancer activity of silver nanoparticles from Panax ginseng fresh leaves in human cancer cells. Biomed Pharmacother 84: $158-165$

14. Lee DG, Lee J, Yang S, Kim K-T, Lee S (2015) Identification of dammarane-type triterpenoid saponins from the roots of Panax ginseng. Nat Prod Sci 21: 111-121

15. Kim DH, Park CH, Park D, Choi YJ, Park MH, Chung KW, Kim SR, Lee JS, Chung HY (2014) Ginsenoside Rc modulates Akt/FoxO1 pathways and suppresses oxidative stress. Arch Pharm Res 37: 813-820

16. Lee DG, Lee J, Kim K-T, Lee S-W, Kim Y-O, Cho I-H, Kim H-J, Park C-G, Lee S (2018) High-performance liquid chromatography analysis of phytosterols in Panax ginseng root grown under different conditions. J Ginseng Res 42: 16-20

17. Ahn SI, Kim SK, Yang BW, Lee ES, Kang CS, Hahm YT (2016) Analysis of ginsenosides and non-saponin components of red ginseng from landraces and new varieties. Korean J Hortic Sci Technol 34: 790_ 798

18. Lee CR, Whang WK, Shin CG, Lee HS, Han ST, Im BO, Ko SK (2004) Comparison of ginsenoside composition and contents in fresh ginseng roots cultivated in Korea, Japan, and China at various ages. Korean J Food Sci Technol 36: 847-850

19. Lee SA, Liuting, Jo HK, Im BO, Cho SH, Whang WK, Ko SK (2010) The comparison of ginsenoside composition contents in notoginseng radix (Sanchi) on various parts and ages. Kor J Pharmacogn 41: 319-322

20. Kim SN, Ha YW, Shin H, Son SH, Wu SJ, Kim YS (2007) Simultaneous quantification of 14 ginsenosides in Panax ginseng C.A. Meyer (Korean red ginseng) by HPLC-ELSD and its application to quality control. J Pharm Biomed Anal 45: 164-170

21. Lee SM (2014) Thermal conversion pathways of ginsenosides in red ginseng processing. Nat Prod Sci 20: 119-125 